\title{
Bayesian Modeling of 3-Component Mixture of Exponentiated Inverted Weibull Distribution under Noninformative Prior
}

\author{
Ammara Nawaz Cheema $\mathbb{D}^{1,2}$ Muhammad Aslam, ${ }^{1}$ Ibrahim M. Almanjahie $\mathbb{D}^{1,4}$ \\ and Ishfaq Ahmad $\mathbb{D}^{5}$ \\ ${ }^{1}$ Department of Mathematics and Statistics, Riphah International University, Islamabad, Pakistan \\ ${ }^{2}$ Department of Mathematics, Air University, Islamabad, Pakistan \\ ${ }^{3}$ Department of Mathematics, College of Science, King Khalid University, Abha 62529, Saudi Arabia \\ ${ }^{4}$ Statistical Research and Studies Support Unit, King Khalid University, Abha 62529, Saudi Arabia \\ ${ }^{5}$ Department of Mathematics and Statistics, Faculty of Basic and Applied Sciences, International Islamic University, \\ Islamabad, Pakistan
}

Correspondence should be addressed to Ammara Nawaz Cheema; ammara.au@gmail.com

Received 26 March 2020; Revised 21 June 2020; Accepted 10 July 2020; Published 29 July 2020

Academic Editor: Luis Payá

Copyright (c) 2020 Ammara Nawaz Cheema et al. This is an open access article distributed under the Creative Commons Attribution License, which permits unrestricted use, distribution, and reproduction in any medium, provided the original work is properly cited.

Bayesian study of 3-component mixture modeling of exponentiated inverted Weibull distribution under right type I censoring technique is conducted in this research work. The posterior distribution of the parameters is obtained assuming the noninformative (Jeffreys and uniform) priors. The different loss functions (squared error, quadratic, precautionary, and DeGroot loss function) are used to obtain the Bayes estimators and posterior risks. The performance of the Bayes estimators through posterior risks under the said loss functions is investigated through simulation process. Real data analysis of tensile strength of carbon fiber is also applied for 3 components to conclude the presentation of Bayes estimators. The limiting expressions are also elaborated for Bayes estimators and posterior risks in this study. The impact of some test termination times and sample sizes is reported on Bayes estimators.

\section{Introduction}

Mixture modeling exists in many situations, particularly whenever we have more than one subpopulation. Mixture densities have beautiful properties to solve the complex problems in an easier manner. Recently, [1] explored 3component mixture modeling of exponentiated Weibull distribution under the Bayesian approach. Exponentiated inverted Weibull distribution (EIWD) has wide application in reliability theory. The authors in [2] used maximum likelihood and Bayes methods to derive parameters of EIWD under the type II censoring scheme. Later on, parameters of EIWD under type II censoring are discussed in [3]. The author in [4] derived Bayes and classical estimators of EIWD using noninformative prior. The authors in [5] proposed 2parameter model of EIWD. Bayesian analysis of shape parameter of EIWD under different loss functions (LFs) is discussed in [6]. Bayes and E-Bayes estimators of EIWD using conjugate prior under different loss functions are estimated in [7]. The authors in [8] studied three-parameter weighted EIWD.

The number of components in a mixture distribution is due to heterogeneity of the parent population. It is often restricted to be finite, although in some cases the component may be infinite. As compared to simple modeling, it provides more attractive description of different statistical frameworks. Mixture modeling has wide applications in survival analysis. Recently, the authors in [9] studied 3-component mixture model of Pareto distribution by using type I right censoring scheme. Bayesian estimation and properties of 3component mixture of Rayleigh distribution are discussed in [10]. The authors in [11] explored 3-component mixture of 
exponential distribution under different loss functions. Moreover, the authors in [12] performed Bayesian estimation for finite mixture of exponential, Rayleigh, and Burr type XII distribution.

Motivated by the abovementioned studies of 3-component mixture and EIWD, we investigate the Bayesian modeling of 3-component mixture of EIWD in this study. The main focus of this paper is to highlight efficient Bayes estimators (BEs) of component and proportional parameter(s). For this reason, two symmetric and two asymmetric LFs are used with noninformative priors, uniform prior (UP), and Jeffreys prior (JP), to obtain such results. The estimators are derived by applying the type I right censoring scheme.

The rest of the paper is designed as follows. In the next section, the 3-component mixture model of EIWD is designed. In Section 3, we illustrate the proposed BEs of different parameters under several LFs. The limiting expressions and simulation study are discussed in Sections 4 and 5. Real data analysis is presented in Section 6. In Section 7, conclusions are provided.

\section{The 3-Component Mixture of the EIWD}

For the shape parameter of a random variable $X$, the pdf (probability density function) with the cdf (cumulative distribution function) of EIWD can be illustrated as

$$
\begin{aligned}
& f\left(x, \theta_{i}\right)=\theta_{i} x^{-2} e^{-\theta_{i} x^{-1}}, \quad \text { where } x \geq 0, \text { and } \theta_{i}>0, i=1, \ldots, 3, \\
& F\left(x, \theta_{i}\right)=e^{-\theta_{i} x^{-1}}, \quad \text { where } x \geq 0, \text { and } \theta_{i}>0, i=1, \ldots, 3 .
\end{aligned}
$$

Here, for EIWD, $\theta_{i}$ is defined as shape parameter.

With $w_{1}$ and $w_{2}$ mixing proportion, a finite 3-component mixture model can be written as

$$
\begin{aligned}
f(x) & =f_{1}(x) w_{1}+f_{2}(x) w_{2}+f_{3}(x)\left(1-w_{1}-w_{2}\right), \quad \text { where } w_{1}, w_{2} \geq 0, \text { and } w_{1}+w_{2} \leq 1, \\
f\left(x ; \theta_{1}, \theta_{2}, \theta_{3}, w_{1}, w_{2}\right) & =w_{1} \theta_{1} x^{-2} e^{-\theta_{1} x^{-1}}+w_{2} \theta_{2} x^{-2} e^{-\theta_{2} x^{-1}}+\left(1-w_{1}-w_{2}\right) \theta_{3} x^{-2} e^{-\theta_{3} x^{-1}}, \quad \text { where } x \geq 0 .
\end{aligned}
$$

For mixing proportion parameters and different component values, a 3-component mixture model of the EIWD is shown in Figure 1.

For 3-component mixture, the cdf is written as

$$
\begin{aligned}
& F(x)=F_{1}(x) w_{1}+F_{2}(x) w_{2}+F_{3}(x)\left(1-w_{1}-w_{2}\right), \\
& F(x)=w_{1} e^{-\theta_{1} x^{-1}}+w_{2} e^{-\theta_{2} x^{-1}}+\left(1-w_{1}-w_{2}\right) e^{-\theta_{3} x^{-1}} .
\end{aligned}
$$

\section{Posterior Distribution Using the Noninformative Priors}

The prior information plays an important role to differentiate between classical and Bayesian inference. The probability distribution which characterizes uncertainty of the parameter, prior to the existing information which is studied, is classified as prior distribution. A prior distribution is differentiated as noninformative prior, if it is smooth comparative towards likelihood function, whereas an informative prior (IP) is defined as a prior that has a contact towards the posterior distribution and is not the subject by the likelihood function. In this section, posterior distributions with the likelihood are obtained assuming the noninformative priors (NIPs) (UP and JP).

3.1. Likelihood Function. Assume that from the 3-component mixture modeling of EIWD $n$ units are consumed in a life assessment process with fixed $t$ (test termination time). Suppose that the selected trial reveals that $n$ units from $r$ failed till fixed $t$ and the $n-r$ where the rest of the units are still in running phase. It is noted that, due to the failures, from $r$ failures, $r_{1}$ failures classified are related to subpopulation I, $r_{2}$ failures belong to subpopulation II, and $r_{3}$ failures are related to subpopulation III. Now, the total uncensored sample points are classified as $r=r_{1}, r_{2}$, and $r_{3}$, whereas the rest of the sample points $n-r$ are considered as censored. Here, we have defined the time to failure, of the $i$ th unit relating to $l$ th subpopulation as $x_{o i}, 0<X_{o i} \leq t$, where $l=1, \ldots, 3$ and $i=1$, $\ldots, r_{1}$, and $t$ is defined as time of test termination.

The likelihood of a 3-component mixture is stated as

$$
L(\Phi \mid x) \propto\{1-F(t)\}^{n-r}\left\{\prod_{i=1}^{r_{1}} w_{1} f_{1}\left(x_{1 i}\right)\right\}\left\{\prod_{i=1}^{r_{2}} w_{2} f_{2}\left(x_{2 i}\right)\right\}\left\{\prod_{i=1}^{r_{3}}\left(1-w_{1}-w_{2}\right) f_{3}\left(x_{3 i}\right)\right\} .
$$




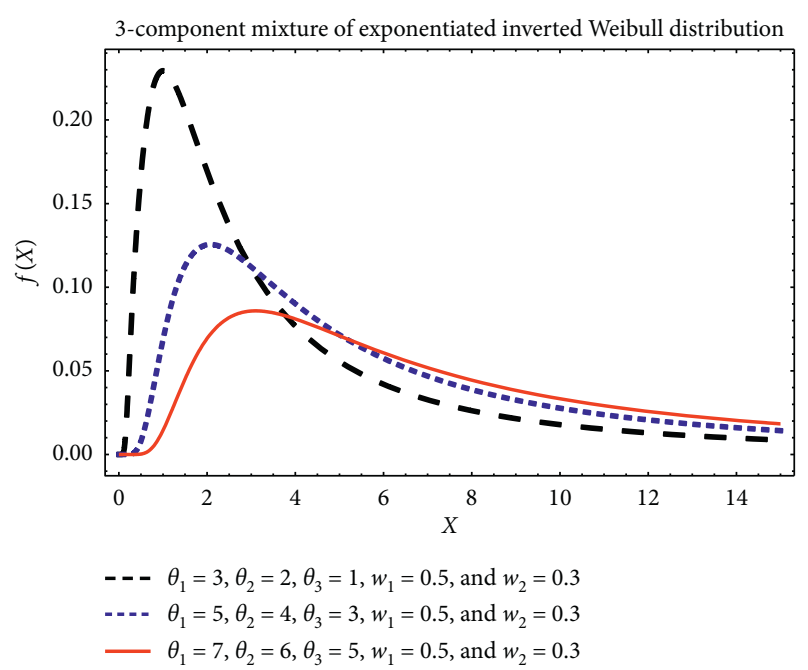

FIgURE 1: The plot of 3-component mixture of EIWD.

By simplifying the expression, we obtain likelihood of 3component mixture model of EIWD as

$L(\Phi \mid x) \propto \mathrm{Se}^{-\theta_{1} B_{11}} e^{-\theta_{2} B_{21}} e^{-\theta_{3} B_{31}} w_{1}^{A_{01}-1} w_{2}^{B_{01}-1} w_{3}^{C_{01}-1} \theta_{1}^{A_{11}-1} \theta_{2}^{A_{21}-1} \theta_{3}^{A_{31}-1}$,

where

$$
\begin{aligned}
A_{11} & =1+r_{1}, \\
A_{21} & =1+r_{2}, \\
A_{31} & =1+r_{3}, \\
B_{11} & =\sum_{k=1}^{r_{1}} x_{1 k}^{-1}+(i-j) t^{-1}, \\
B_{21}= & \sum_{k=1}^{r_{2}} x_{2 k}^{-1}+(j-l) t^{-1}, \\
B_{31}= & \sum_{k=1}^{r_{3}} x_{3 k}^{-1}+l t^{-1}, \\
A_{01}= & r_{1}+1+i-j, \\
B_{01}= & r_{2}+1+j-l, \\
C_{01}= & r_{3}+1+l, \\
w_{3} & =1-w_{1}-w_{2}, \\
S & =\sum_{i=0}^{n-r} \sum_{j=0}^{i} \sum_{l=0}^{j}(-1)^{n-r}\left(\begin{array}{c}
n-r \\
i
\end{array}\right)\left(\begin{array}{c}
i \\
j
\end{array}\right)\left(\begin{array}{l}
j \\
l
\end{array}\right),
\end{aligned}
$$

and $x=\left(x_{11}, \ldots, x_{1 r_{1}}, x_{21}, \ldots, x_{2 r_{2}}, x_{31}, \ldots, x_{3 r_{3}}\right)$ for the uncensored observations are the failure times and $\Phi=\left(\theta_{1}, \theta_{2}, \theta_{3}, w_{1}, w_{2}\right)$.

3.2. Posterior Distribution Using Uniform Prior (UP). The UP and JP are the most applied NIPs. Most of the researchers mentioned that UP is the most applied prior, for the evaluation, of required parameters of concern (see [13-15]). The improper UP for the parametric component part of EIWD $\theta_{1}, \theta_{2}$, and $\theta_{3}$, i.e., $\theta_{1} \sim U(0, \infty), \theta_{2} \sim U(0, \infty)$, and $\theta_{3} \sim U(0, \infty)$, is considered. The UP in the interval $(0,1)$ is applied for the parameters $w_{1}$ and $w_{2}$, that is, $w_{1} \sim U(0,1)$ and $w_{2} \sim U(0,1)$. The joint prior distribution of parameters $\theta_{1}, \theta_{2}, \theta_{3}, w_{1}$, and $w_{2}$ can be stated as

$$
\begin{aligned}
\pi_{1}(\Phi) & =1 ; \\
\theta_{1}, \theta_{2} \text { and } \theta_{3} & >0 ; \\
w_{1} \text { and } w_{2} & \geq 0 ; \\
w_{1}+w_{2} & \leq 1 .
\end{aligned}
$$

So, under the UP, the joint posterior distribution of parameters $\theta_{1}, \theta_{2}, \theta_{3}, w_{1}$, and $w_{2}$ can be written as

$$
\begin{aligned}
g_{1}(\Phi \mid x)= & \Omega_{1}^{-1} S \theta_{1}^{A_{11}-1} \theta_{2}^{A_{21}-1} \theta_{3}^{A_{31}-1} e^{-\theta_{1} B_{11}} e^{-\theta_{2} B_{21}} e^{-\theta_{3} B_{31}} \\
& \cdot w_{1}^{A_{01}-1} w_{2}^{B_{01}-1} w_{3}^{C_{01}-1}
\end{aligned}
$$

where

$$
\begin{aligned}
\Omega_{1}= & \Gamma\left(A_{31}\right) \Gamma\left(A_{21}\right) \Gamma\left(A_{11}\right) \sum_{i=0}^{n-r} \sum_{j=0}^{i} \sum_{l=0}^{j}(-1)^{n-r}\left(\begin{array}{c}
n-r \\
i
\end{array}\right) \\
& \cdot\left(\begin{array}{c}
i \\
j
\end{array}\right)\left(\begin{array}{l}
j \\
l
\end{array}\right) B\left(A_{01}, B_{01}, C_{01}\right) B_{11}^{-A_{11}} B_{21}^{-A_{21}} B_{31}^{-A_{31}},
\end{aligned}
$$

and $B\left(A_{01}, B_{01}, C_{01}\right)$ is the beta function and extended as $B\left(B_{01}, C_{01}\right) B\left(A_{01}, B_{01}+C_{01}\right)$.

3.3. Posterior Distribution Using the Jeffreys Prior (JP). The Jeffreys proposed a rule of thumb to specify noninformative prior for parameter $\theta$ as follows: If $\theta \in[a, b]$, then, $g(\theta)=$ constant, and if $\theta \in[0, \infty]$, then $g(\theta)=(1 / \theta)$, using transformation of the variables (see [16-19]). In respect of proportion parameters $w_{1}$ and $w_{2}$, the JP is used as $w_{1} \sim U(0,1)$ and $w_{2} \sim U(0,1)$. The joint prior distribution of parameters, with the assumption of independence of the said parameters $\theta_{1}, \theta_{2}, \theta_{3}, w_{1}$, and $w_{2}$, is stated as

$$
\pi_{2}(\Phi) \propto \frac{1}{\theta_{1} \theta_{2} \theta_{3}}, \quad \theta_{1} \theta_{2} \theta_{3}>0, w_{1}, w_{2} \geq 0, w_{1}+w_{2} \leq 1 .
$$

Then, the joint posterior distribution becomes

$$
\begin{aligned}
g_{2}(\Phi \mid x)= & \Omega_{2}^{-1} S \theta_{1}^{A_{12}} \theta_{2}^{A_{22}} \theta_{3}^{A_{32}} e^{-\theta_{1} B_{12}} e^{-\theta_{2} B_{22}} e^{-\theta_{3} B_{32}} w_{1}^{A_{02}-1} \\
& \cdot w_{2}^{B_{02}-1} w_{3}^{C_{02}-1}
\end{aligned}
$$

where 


$$
\begin{aligned}
& A_{12}=-2+r_{1}, \\
& A_{22}=-2+r_{2}, \\
& A_{32}=-2+r_{3}, \\
& B_{12}=(i-j) t^{-1}+\sum_{k=1}^{r_{1}} x_{1 k}^{-1}+, \\
& B_{22}=(j-l) t^{-1}+\sum_{k=1}^{r_{2}} x_{2 k}^{-1}, \\
& B_{32}=l t^{-1}+\sum_{k=1}^{r_{3}} x_{3 k}^{-1}, \\
& A_{02}=r_{1}+i+1-j, \\
& B_{02}=r_{2}+1+j-l, \\
& C_{02}=r_{3}+1+l, \\
& w_{3}=1-w_{1}-w_{2}, \\
& \Omega_{2}=\Gamma\left(A_{32}\right) \Gamma\left(A_{22}\right) \Gamma\left(A_{12}\right) \sum_{i=0}^{n-r} \sum_{j=0}^{i} \sum_{l=0}^{j}(-1)^{n-r}\left(\begin{array}{c}
n-r \\
i
\end{array}\right)\left(\begin{array}{l}
i \\
j
\end{array}\right)\left(\begin{array}{l}
j \\
l
\end{array}\right) B\left(A_{02}, B_{02}, C_{02}\right) B_{12}^{-A_{12}} B_{22}^{-A_{22}} B_{32}^{-A_{32}} .
\end{aligned}
$$

3.4. BEs and PRs under LFs. The real-valued function which illustrates a loss for estimator over the exact value of parameter is defined as loss function (LF). The current section discussed BEs and posterior risks (PRs) over four different LFs, that is, squared error loss function (SELF), quadratic loss function (QLF), precautionary loss function (PLF), and DeGroot loss function (DLF).
3.4.1. BEs and PRs among SELF. For $d$ which is BE, $\operatorname{PR} \rho(d)$ can be written as $\rho(d)=E\{L(\beta, d)\}$, where $L(\beta, d)=(d-\beta)^{T}(d-\beta)$ is the SELF.

We obtain BEs and PRs assuming NIPs for the component and proportion parameters $\theta_{1}, \theta_{2}, \theta_{3}, w_{1}$, and $w_{2}$ under SELF as

$$
\begin{aligned}
\hat{\theta}_{1 v} & =\Omega_{v}^{-1} \Gamma\left(A_{3 v}\right) \Gamma\left(A_{1 v}+1\right) \Gamma\left(A_{2 v}\right) S \beta\left(A_{0 v}, B_{0 v}+C_{0 v}\right) B\left(B_{0 v}, C_{0 v}\right) B_{1 v}^{-\left(A_{1 v}+1\right)} B_{2 v}^{-A_{2 v}} B_{3 v}^{-A_{3 v}}, \\
\hat{\theta}_{2 v} & =\Omega_{v}^{-1} \Gamma\left(A_{3 v}\right) \Gamma\left(A_{1 v}\right) \Gamma\left(A_{2 v}+1\right) S \beta\left(A_{0 v}, B_{0 v}+C_{0 v}\right) B\left(B_{0 v}, C_{0 v}\right) B_{1 v}^{-A_{1 v}} B_{2 v}^{-\left(A_{2 v}+1\right)} B_{3 v}^{-A_{3 v}}, \\
\hat{\theta}_{3 v} & =\Omega_{v}^{-1} \Gamma\left(A_{1 v}\right) \Gamma\left(A_{2 v}\right) \Gamma\left(A_{3 v}+1\right) S \beta\left(A_{0 v}, B_{0 v}+C_{0 v}\right) B\left(B_{0 v}, C_{0 v}\right) B_{1 v}^{-A_{1 v}} B_{2 v}^{-A_{2 v}} B_{3 v}^{-\left(A_{3 v}+1\right)}, \\
\hat{w}_{1 v} & =\Omega_{v}^{-1} \Gamma\left(A_{3 v}\right) \Gamma\left(A_{1 v}\right) \Gamma\left(A_{2 v}\right) S \beta\left(A_{0 v}+1, B_{0 v}+C_{0 v}\right) B\left(B_{0 v}, C_{0 v}\right) B_{1 v}^{-A_{1 v}} B_{2 v}^{-A_{2 v}} B_{3 v}^{-A_{3 v}}, \\
\hat{w}_{2 v} & =\Omega_{v}^{-1} \Gamma\left(A_{3 v}\right) \Gamma\left(A_{1 v}\right) \Gamma\left(A_{2 v}\right) S \beta\left(B_{0 v}+1, A_{0 v}+C_{0 v}\right) B\left(A_{0 v}, C_{0 v}\right) B_{1 v}^{-A_{1 v}} B_{2 v}^{-A_{2 v}} B_{3 v}^{-A_{3 v}}, \\
\rho\left(\widehat{\theta}_{1 v}\right) & =\Gamma\left(A_{3 v}\right) \Gamma\left(A_{1 v}+2\right) \Gamma\left(A_{2 v}\right) S B_{3 v}^{-A_{3 v}} B_{1 v}^{-A_{1 v}-2} B_{2 v}^{-A_{2 v}} B\left(A_{0 v}, B_{0 v}+C_{0 v}\right) B\left(B_{0 v}, C_{0 v}\right)-\widehat{\theta}_{1 v}^{2}, \\
\rho\left(\hat{\theta}_{2 v}\right) & =\Gamma\left(A_{3 v}\right) \Gamma\left(A_{1 v}\right) \Gamma\left(A_{2 v}+2\right) S B_{3 v}^{-A_{3 v}} B_{1 v}^{-A_{1 v}} B_{2 v}^{-A_{2 v}-2} B\left(A_{0 v}, B_{0 v}+C_{0 v}\right) B\left(B_{0 v}, C_{0 v}\right)-\widehat{\theta}_{2 v}^{2}, \\
\rho\left(\hat{\theta}_{3 v}\right) & =\Gamma\left(A_{3 v}+2\right) \Gamma\left(A_{1 v}\right) \Gamma\left(A_{2 v}\right) S B_{3 v}^{-A_{3 v}-2} B_{1 v}^{-A_{1 v}} B_{2 v}^{-A_{2 v}} B\left(A_{0 v}, B_{0 v}+C_{0 v}\right) B\left(B_{0 v}, C_{0 v}\right)-\widehat{\theta}_{3 v}^{2}, \\
\rho\left(\widehat{w}_{1 v}\right) & =\Gamma\left(A_{3 v}\right) \Gamma\left(A_{1 v}\right) \Gamma\left(A_{2 v}\right) S B_{3 v}^{-A_{3 v}} B_{1 v}^{-A_{1 v}} B_{2 v}^{-A_{2 v}} B\left(A_{0 v}+2, B_{0 v}+C_{0 v}\right) B\left(B_{0 v}, C_{0 v}\right)-\widehat{w}_{1 v}^{2}, \\
\rho\left(\widehat{w}_{2 v}\right) & =\Gamma\left(A_{3 v}\right) \Gamma\left(A_{1 v}\right) \Gamma\left(A_{2 v}\right) S B_{3 v}^{-A_{3 v}} B_{1 v}^{-A_{1 v}} B_{2 v}^{-A_{2 v}} B\left(B_{0 v}+2, A_{0 v}+C_{0 v}\right) B\left(A_{0 v}, C_{0 v}\right)-\widehat{w}_{2 v}^{2},
\end{aligned}
$$


where $v=1$ for the UP and $v=2$ for the JP.

3.4.2. BEs and PRs Assuming the UP and the JP under QLF. QLF is symmetric LF and mostly used in the least square theory. It needs more care to tackle due to its variance properties and being symmetric, where $L(\beta, d)=\alpha(\beta-d)^{2}$ is the QLF. We can, respectively, define the BE and the PR under QLF as

$$
\frac{E\left(\beta^{-1}\right)}{E\left(\beta^{-2}\right)}, \quad 1-\frac{\left\{E\left(\beta^{-1}\right)\right\}^{2}}{E\left(\beta^{-2}\right)} .
$$

By applying this concept using GP and ILP, the derivation of BEs and PRs is

$$
\begin{aligned}
& \widehat{\theta}_{1 v}=\frac{\Gamma\left(A_{2 v}\right) \Gamma\left(A_{3 v}\right) \Gamma\left(A_{1 v}-1\right) S B_{3 v}^{-A_{3 v}} B_{1 v}^{-\left(A_{1 v}-1\right)} B_{2 v}^{-A_{2 v}} B\left(A_{0 v}, B_{0 v}+C_{0 v}\right) B\left(B_{0 v}, C_{0 v}\right)}{\Gamma\left(A_{2 v}\right) \Gamma\left(A_{3 v}\right) \Gamma\left(A_{1 v}-2\right) S B_{3 v}^{-A_{3 v}} B_{1 v}^{-A_{1 v}+2} B_{2 v}^{-A_{2 v}} B\left(A_{0 v}, B_{0 v}+C_{0 v}\right) B\left(B_{0 v}, C_{0 v}\right)}, \\
& \widehat{\theta}_{2 v}=\frac{\Gamma\left(A_{2 v}-1\right) \Gamma\left(A_{3 v}\right) \Gamma\left(A_{1 v}\right) S B_{2 v}^{-\left(A_{2 v}-1\right)} B_{1 v}^{-A_{1 v}} B_{3 v}^{-A_{3 v}} B\left(A_{0 v}, B_{0 v}+C_{0 v}\right) B\left(B_{0 v}, C_{0 v}\right)}{\Gamma\left(A_{2 v}-2\right) \Gamma\left(A_{3 v}\right) \Gamma\left(A_{1 v}\right) S B_{2 v}^{-A_{2 v}+2} B_{1 v}^{-A_{1 v}} B_{3 v}^{-A_{3 v}} B\left(A_{0 v}, B_{0 v}+C_{0 v}\right) B\left(B_{0 v}, C_{0 v}\right)}, \\
& \widehat{\theta}_{3 v}=\frac{\Gamma\left(A_{2 v}\right) \Gamma\left(A_{3 v}-1\right) \Gamma\left(A_{1 v}\right) S B_{1 v}^{-A_{1 v}} B_{2 v}^{-A_{2 v}} B_{3 v}^{-\left(A_{3 v}-1\right)} B\left(A_{0 v}, B_{0 v}+C_{0 v}\right) B\left(B_{0 v}, C_{0 v}\right)}{\Gamma\left(A_{2 v}\right) \Gamma\left(A_{3 v}-2\right) \Gamma\left(A_{1 v}\right) S B_{1 v}^{-A_{1 v}} B_{2 v}^{-A_{2 v}} B_{3 v}^{-\left(A_{3 v}-2\right)} B\left(A_{0 v}, B_{0 v}+C_{0 v}\right) B\left(B_{0 v}, C_{0 v}\right)}, \\
& \widehat{w}_{1 v}=\frac{S B_{1 v}^{-A_{1 v}} B_{2 v}^{-A_{2 v}} B_{3 v}^{-A_{3 v}} B\left(A_{0 v}-1, B_{0 v}+C_{0 v}\right) B\left(B_{0 v}, C_{0 v}\right)}{S B_{1 v}^{-A_{1 v}} B_{2 v}^{-A_{2 v}} B_{3 v}^{-A_{3 v}} B\left(A_{0 v}-2, B_{0 v}+C_{0 v}\right) B\left(B_{0 v}, C_{0 v}\right)}, \\
& \widehat{w}_{2 v}=\frac{S B_{1 v}^{-A_{1 v}} B_{2 v}^{-A_{2 v}} B_{3 v}^{-A_{3 v}} B\left(B_{0 v}-1, A_{0 v}+C_{0 v}\right) B\left(A_{0 v}, C_{0 v}\right)}{S B_{1 v}^{-A_{1 v}} B_{2 v}^{-A_{2 v}} B_{3 v}^{-A_{3 v}} B\left(B_{0 v}-2, A_{0 v}+C_{0 v}\right) B\left(A_{0 v}, C_{0 v}\right)}, \\
& \rho\left(\widehat{\theta}_{1 v}\right)=1-\frac{\left\{\Gamma\left(A_{1 v}-1\right) \Gamma\left(A_{2 v}\right) \Gamma\left(A_{3 v}\right) S B_{3 v}^{-A_{3 v}} B_{1 v}^{-\left(A_{1 v}-1\right)} B_{2 v}^{-A_{2 v}} B\left(A_{0 v}, B_{0 v}+C_{0 v}\right) B\left(B_{0 v}, C_{0 v}\right)\right\}^{2}}{\Gamma\left(A_{1 v}-2\right) \Gamma\left(A_{2 v}\right) \Gamma\left(A_{3 v}\right) S B_{3 v}^{-A_{3 v}} B_{1 v}^{-\left(A_{1 v}-2\right)} B_{2 v}^{-A_{2 v}} B\left(A_{0 v}, B_{0 v}+C_{0 v}\right) B\left(B_{0 v}, C_{0 v}\right)}, \\
& \rho\left(\widehat{\theta}_{2 v}\right)=1-\frac{\left\{\Gamma\left(A_{1 v}\right) \Gamma\left(A_{2 v}-1\right) \Gamma\left(A_{3 v}\right) S B_{3 v}^{-A_{3 v}} B_{1 v}^{-A_{1 v}} B_{2 v}^{-\left(A_{2 v}-1\right)} B\left(A_{0 v}, B_{0 v}+C_{0 v}\right) B\left(B_{0 v}, C_{0 v}\right)\right\}^{2}}{\Gamma\left(A_{1 v}\right) \Gamma\left(A_{2 v}-2\right) \Gamma\left(A_{3 v}\right) S B_{3 v}^{-A_{3 v}} B_{1 v}^{-A_{1 v}} B_{2 v}^{-\left(A_{2 v}-2\right)} B\left(A_{0 v}, B_{0 v}+C_{0 v}\right) B\left(B_{0 v}, C_{0 v}\right)}, \\
& \rho\left(\widehat{\theta}_{3 v}\right)=1-\frac{\left\{\Gamma\left(A_{1 v}\right) \Gamma\left(A_{2 v}\right) \Gamma\left(A_{3 v}-1\right) S B_{3 v}^{-\left(A_{3 v}-1\right)} B_{1 v}^{-A_{1 v}} B_{2 v}^{-A_{2 v}} B\left(A_{0 v}, B_{0 v}+C_{0 v}\right) B\left(B_{0 v}, C_{0 v}\right)\right\}^{2}}{\Gamma\left(A_{1 v}\right) \Gamma\left(A_{2 v}\right) \Gamma\left(A_{3 v}-2\right) S B_{3 v}^{-\left(A_{3 v}-2\right)} B_{1 v}^{-A_{1 v}} B_{2 v}^{-A_{2 v}} B\left(A_{0 v}, B_{0 v}+C_{0 v}\right) B\left(B_{0 v}, C_{0 v}\right)}, \\
& \rho\left(\widehat{w}_{1 v}\right)=1-\frac{\left\{\Gamma\left(A_{1 v}\right) \Gamma\left(A_{2 v}\right) \Gamma\left(A_{3 v}\right) S B_{3 v}^{-A_{3 v}} B_{1 v}^{-A_{1 v}} B_{2 v}^{-A_{2 v}} B\left(A_{0 v}-1, B_{0 v}+C_{0 v}\right) B\left(B_{0 v}, C_{0 v}\right)\right\}^{2}}{\Gamma\left(A_{1 v}\right) \Gamma\left(A_{2 v}\right) \Gamma\left(A_{3 v}\right) S B_{3 v}^{-A_{3 v}} B_{1 v}^{-A_{1 v}} B_{2 v}^{-A_{2 v}} B\left(A_{0 v}-2, B_{0 v}+C_{0 v}\right) B\left(B_{0 v}, C_{0 v}\right)}, \\
& \rho\left(\widehat{w}_{2 v}\right)=1-\frac{\left\{\Gamma\left(A_{1 v}\right) \Gamma\left(A_{2 v}\right) \Gamma\left(A_{3 v}\right) S B_{3 v}^{-A_{3 v}} B_{1 v}^{-A_{1 v}} B_{2 v}^{-A_{2 v}} B\left(B_{0 v}-1, A_{0 v}+C_{0 v}\right) B\left(A_{0 v}, C_{0 v}\right)\right\}^{2}}{\Gamma\left(A_{1 v}\right) \Gamma\left(A_{2 v}\right) \Gamma\left(A_{3 v}\right) S B_{3 v}^{-A_{3 v}} B_{1 v}^{-A_{1 v}} B_{2 v}^{-A_{2 v}} B\left(B_{0 v}-2, A_{0 v}+C_{0 v}\right) B\left(A_{0 v}, C_{0 v}\right)},
\end{aligned}
$$

where $v=1$ and $v=2$ for the UP and JP.

3.4.3. BEs and PRs for the UP and the JP among PLF. PLF is an asymmetric LF, which firstly introduced by [20]. The general form of PLFs which is also a special case can be stated as $L(\beta, d)=\left(\left((\beta-d)^{2}\right) / d\right)$. Under PLF, the derivation of BEs and PRs can be obtained with the help of

$$
\begin{aligned}
d & =\left\{E_{(\beta \mid x)}\left(\beta^{2}\right)\right\}^{1 / 2}, \\
\rho(d) & =2\left\{E_{(\beta \mid x)}\left(\beta^{2}\right)\right\}^{1 / 2}-2 E_{(\beta \mid x)}(\beta) .
\end{aligned}
$$


The derived results for BEs and PRs under the said priors and LF are observed as

$$
\begin{aligned}
\widehat{\theta}_{1 v} & =\left\{\Gamma\left(A_{1 v}+2\right) \Gamma\left(A_{2 v}\right) \Gamma\left(A_{3 v}\right) S B_{3 v}^{-A_{3 v}} B_{1 v}^{-\left(A_{1 v}+2\right)} B_{2 v}^{-A_{2 v}} B\left(A_{0 v}, B_{0 v}+C_{0 v}\right) B\left(B_{0 v}, C_{0 v}\right)\right\}^{1 / 2}, \\
\widehat{\theta}_{2 v} & =\left\{\Gamma\left(A_{1 v}\right) \Gamma\left(A_{2 v}+2\right) \Gamma\left(A_{3 v}\right) S B_{3 v}^{-A_{3 v}} B_{1 v}^{-A_{1 v}} B_{2 v}^{-\left(A_{2 v}+2\right)} B\left(A_{0 v}, B_{0 v}+C_{0 v}\right) B\left(B_{0 v}, C_{0 v}\right)\right\}^{1 / 2}, \\
\widehat{\theta}_{3 v} & =\left\{\Gamma\left(A_{1 v}\right) \Gamma\left(A_{2 v}\right) \Gamma\left(A_{3 v}+2\right) S B_{3 v}^{-\left(A_{3 v}+2\right)} B_{1 v}^{-A_{1 v}} B_{2 v}^{-A_{2 v}} B\left(A_{0 v}, B_{0 v}+C_{0 v}\right) B\left(B_{0 v}, C_{0 v}\right)\right\}^{1 / 2}, \\
\widehat{w}_{1 v} & =\left\{\Gamma\left(A_{1 v}\right) \Gamma\left(A_{2 v}\right) \Gamma\left(A_{3 v}\right) S B_{3 v}^{-A_{3 v}} B_{1 v}^{-A_{1 v}} B_{2 v}^{-A_{2 v}} B\left(A_{0 v}+2, B_{0 v}+C_{0 v}\right) B\left(B_{0 v}, C_{0 v}\right)\right\}^{1 / 2}, \\
\widehat{w}_{2 v} & =\left\{\Gamma\left(A_{1 v}\right) \Gamma\left(A_{2 v}\right) \Gamma\left(A_{3 v}\right) S B_{3 v}^{-A_{3 v}} B_{1 v}^{-A_{1 v}} B_{2 v}^{-A_{2 v}} B\left(B_{0 v}+2, A_{0 v}+C_{0 v}\right) B\left(A_{0 v}, C_{0 v}\right)\right\}^{1 / 2}, \\
\rho\left(\widehat{\theta}_{1 v}\right) & =2\left\{\widehat{\theta}_{1 v}-\Gamma\left(A_{1 v}+1\right) \Gamma\left(A_{2 v}\right) \Gamma\left(A_{3 v}\right) S B_{3 v}^{-A_{3 v}} B_{1 v}^{-\left(A_{1 v}+1\right)} B_{2 v}^{-A_{2 v}} B\left(A_{0 v}, B_{0 v}+C_{0 v}\right) B\left(B_{0 v}, C_{0 v}\right)\right\}, \\
\rho\left(\widehat{\theta}_{2 v}\right) & =2\left\{\hat{\theta}_{2 v}-\Gamma\left(A_{1 v}\right) \Gamma\left(A_{2 v}+1\right) \Gamma\left(A_{3 v}\right) S B_{3 v}^{-A_{3 v}} B_{1 v}^{-A_{1 v}} B_{2 v}^{-\left(A_{2 v}+1\right)} B\left(A_{0 v}, B_{0 v}+C_{0 v}\right) B\left(B_{0 v}, C_{0 v}\right)\right\}, \\
\rho\left(\widehat{\theta}_{3 v}\right) & =2\left\{\widehat{\theta}_{3 v}-\Gamma\left(A_{1 v}\right) \Gamma\left(A_{2 v}\right) \Gamma\left(A_{3 v}+1\right) S B_{3 v}^{-\left(A_{3 v}+1\right)} B_{1 v}^{-A_{1 v}} B_{2 v}^{-A_{2 v}} B\left(A_{0 v}, B_{0 v}+C_{0 v}\right) B\left(B_{0 v}, C_{0 v}\right)\right\}, \\
\rho\left(\widehat{w}_{1 v}\right) & =2\left\{\widehat{w}_{1 v}-\Gamma\left(A_{1 v}\right) \Gamma\left(A_{2 v}\right) \Gamma\left(A_{3 v}\right) S B_{3 v}^{-A_{3 v}} B_{1 v}^{-A_{1 v}} B_{2 v}^{-A_{2 v}} B\left(A_{0 v}+1, B_{0 v}+C_{0 v}\right) B\left(B_{0 v}, C_{0 v}\right)\right\}, \\
\rho\left(\widehat{w}_{2 v}\right) & =2\left\{\widehat{w}_{2 v}-\Gamma\left(A_{1 v}\right) \Gamma\left(A_{2 v}\right) \Gamma\left(A_{3 v}\right) S B_{3 v}^{-A_{3 v}} B_{1 v}^{-A_{1 v}} B_{2 v}^{-A_{2 v}} B\left(B_{0 v}+1, A_{0 v}+C_{0 v}\right) B\left(A_{0 v}, C_{0 v}\right)\right\},
\end{aligned}
$$

where $v=1$ for the UP and $v=2$ for the JP, respectively.

The derived BEs and PRs using the assumed priors are

3.4.4. BEs and PRs Assuming the UP and the JP under DLF. Another asymmetric LF introduced by [21] is DLF, written as $L(\beta, d)=((\beta-d) / d)^{2}$. The BEs and the PRs among DLF are obtained as

$$
\begin{gathered}
d=\frac{\left\{E_{(\beta \mid x)}(\beta)\right\}^{2}}{E_{(\beta \mid x)}(\beta)}, \\
\rho(d)=1-\frac{\left\{E_{(\beta \mid x)}(\beta)\right\}^{2}}{E_{(\beta \mid x)}\left(\beta^{2}\right)} .
\end{gathered}
$$

$$
\begin{gathered}
\widehat{\theta}_{1 v}=\frac{\Gamma\left(A_{3 v}\right) \Gamma\left(A_{1 v}+2\right) \Gamma\left(A_{2 v}\right) S B_{1 v}^{-\left(A_{1 v}+2\right)} B_{2 v}^{-A_{2 v}} B_{3 v}^{-A_{3 v}} B\left(A_{0 v}, B_{0 v}+C_{0 v}\right) B\left(B_{0 v}, C_{0 v}\right)}{\Gamma\left(A_{3 v}\right) \Gamma\left(A_{1 v}+1\right) \Gamma\left(A_{2 v}\right) S B_{1 v}^{-\left(A_{1 v}+1\right)} B_{2 v}^{-A_{2 v}} B_{3 v}^{-A_{3 v}} B\left(A_{0 v}, B_{0 v}+C_{0 v}\right) B\left(B_{0 v}, C_{0 v}\right)}, \\
\widehat{\theta}_{2 v}=\frac{\Gamma\left(A_{3 v}\right) \Gamma\left(A_{1 v}\right) \Gamma\left(A_{2 v}+2\right) S B_{1 v}^{-A_{1 v}} B_{2 v}^{-\left(A_{2 v}+2\right)} B_{3 v}^{-A_{3 v}} B\left(A_{0 v}, B_{0 v}+C_{0 v}\right) B\left(B_{0 v}, C_{0 v}\right)}{\Gamma\left(A_{3 v}\right) \Gamma\left(A_{1 v}\right) \Gamma\left(A_{2 v}+1\right) S B_{1 v}^{-A_{1 v}} B_{2 v}^{-\left(A_{2 v}+1\right)} B_{3 v}^{-A_{3 v}} B\left(A_{0 v}, B_{0 v}+C_{0 v}\right) B\left(B_{0 v}, C_{0 v}\right)}, \\
\widehat{\theta}_{3 v}=\frac{\Gamma\left(A_{3 v}+2\right) \Gamma\left(A_{1 v}\right) \Gamma\left(A_{2 v}\right) S B_{1 v}^{-A_{1 v}} B_{2 v}^{-A_{2 v}} B_{3 v}^{-\left(A_{3 v}+2\right)} B\left(A_{0 v}, B_{0 v}+C_{0 v}\right) B\left(B_{0 v}, C_{0 v}\right)}{\Gamma\left(A_{3 v}+1\right) \Gamma\left(A_{1 v}\right) \Gamma\left(A_{2 v}\right) S B_{1 v}^{-A_{1 v}} B_{2 v}^{-A_{2 v}} B_{3 v}^{-\left(A_{3 v}+1\right)} B\left(A_{0 v}, B_{0 v}+C_{0 v}\right) B\left(B_{0 v}, C_{0 v}\right)},
\end{gathered}
$$




$$
\begin{aligned}
\widehat{w}_{1 v}= & \frac{S B_{1 v}^{-A_{1 v}} B_{2 v}^{-A_{2 v}} B_{3 v}^{-A_{3 v}} B\left(A_{0 v}+2, B_{0 v}+C_{0 v}\right) B\left(B_{0 v}, C_{0 v}\right)}{S B_{1 v}^{-A_{1 v}} B_{2 v}^{-A_{2 v}} B_{3 v}^{-A_{3 v}} B\left(A_{0 v}+1, B_{0 v}+C_{0 v}\right) B\left(B_{0 v}, C_{0 v}\right)}, \\
\widehat{w}_{2 v}= & \frac{S B_{1 v}^{-A_{1 v}} B_{2 v}^{-A_{2 v}} B_{3 v}^{-A_{3 v}} B\left(B_{0 v}+2, A_{0 v}+C_{0 v}\right) B\left(A_{0 v}, C_{0 v}\right)}{S B_{1 v}^{-A_{1 v}} B_{2 v}^{-A_{2 v}} B_{3 v}^{-A_{3 v}} B\left(B_{0 v}+1, A_{0 v}+C_{0 v}\right) B\left(A_{0 v}, C_{0 v}\right)}, \\
\rho\left(\widehat{\theta}_{1 v}\right)= & 1-\frac{\left\{\Gamma\left(A_{1 v}+1\right) \Gamma\left(A_{2 v}\right) \Gamma\left(A_{3 v}\right) S B_{3 v}^{-A_{3 v}} B_{1 v}^{-\left(A_{1 v}+1\right)} B_{2 v}^{-A_{2 v}} B\left(A_{0 v}, B_{0 v}+C_{0 v}\right) B\left(B_{0 v}, C_{0 v}\right)\right\}^{2}}{\Gamma\left(A_{1 v}+2\right) \Gamma\left(A_{2 v}\right) \Gamma\left(A_{3 v}\right) S B_{3 v}^{-A_{3 v}} B_{1 v}^{-\left(A_{1 v}+2\right)} B_{2 v}^{-A_{2 v}} B\left(A_{0 v}, B_{0 v}+C_{0 v}\right) B\left(B_{0 v}, C_{0 v}\right)}, \\
\rho\left(\widehat{\theta}_{2 v}\right)= & 1-\frac{\left\{\Gamma\left(A_{1 v}\right) \Gamma\left(A_{2 v}+1\right) \Gamma\left(A_{3 v}\right) S B_{3 v}^{-A_{3 v}} B_{1 v}^{-A_{1 v}} B_{2 v}^{-\left(A_{2 v}+1\right)} B\left(A_{0 v}, B_{0 v}+C_{0 v}\right) B\left(B_{0 v}, C_{0 v}\right)\right\}^{2}}{\Gamma\left(A_{1 v}\right) \Gamma\left(A_{2 v}+2\right) \Gamma\left(A_{3 v}\right) S B_{3 v}^{-A_{3 v}} B_{1 v}^{-A_{1 v}} B_{2 v}^{-\left(A_{2 v}+2\right)} B\left(A_{0 v}, B_{0 v}+C_{0 v}\right) B\left(B_{0 v}, C_{0 v}\right)}, \\
\rho\left(\widehat{\theta}_{3 v}\right)= & 1-\frac{\left\{\Gamma\left(A_{3 v}+1\right) \Gamma\left(A_{1 v}\right) \Gamma\left(A_{2 v}\right) S B_{3 v}^{-\left(A_{3 v}+1\right)} B_{1 v}^{-A_{1 v}} B_{2 v}^{-A_{2 v}} B\left(A_{0 v}, B_{0 v}+C_{0 v}\right) B\left(B_{0 v}, C_{0 v}\right)\right\}^{2}}{\Gamma\left(A_{3 v}+2\right) \Gamma\left(A_{1 v}\right) \Gamma\left(A_{2 v}\right) S B_{3 v}^{-\left(A_{3 v}+2\right)} B_{1 v}^{-A_{1 v}} B_{2 v}^{-A_{2 v}} B\left(A_{0 v}, B_{0 v}+C_{0 v}\right) B\left(B_{0 v}, C_{0 v}\right)}, \\
\rho\left(\widehat{w}_{1 v}\right)= & 1-\frac{\left\{\Gamma\left(A_{1 v}\right) \Gamma\left(A_{2 v}\right) \Gamma\left(A_{3 v}\right) S B_{3 v}^{-A_{3 v}} B_{1 v}^{-A_{1 v}} B_{2 v}^{-A_{2 v}} B\left(A_{0 v}+1, B_{0 v}+C_{0 v}\right) B\left(B_{0 v}, C_{0 v}\right)\right\}^{2}}{\Gamma\left(A_{3 v}\right) \Gamma\left(A_{1 v}\right) \Gamma\left(A_{2 v}\right) S B_{3 v}^{-A_{3 v}} B_{1 v}^{-A_{1 v}} B_{2 v}^{-A_{2 v}} B\left(A_{0 v}+2, B_{0 v}+C_{0 v}\right) B\left(B_{0 v}, C_{0 v}\right)}, \\
\rho\left(\widehat{w}_{2 v}\right)= & 1-\frac{\left\{\Gamma\left(A_{1 v}\right) \Gamma\left(A_{2 v}\right) \Gamma\left(A_{3 v}\right) S B_{3 v}^{-A_{3 v}} B_{1 v}^{-A_{1 v}} B_{2 v}^{-A_{2 v}} B\left(B_{0 v}+1, A_{0 v}+C_{0 v}\right) B\left(A_{0 v}, C_{0 v}\right)\right\}^{2}}{\Gamma\left(A_{3 v}\right) \Gamma\left(A_{1 v}\right) \Gamma\left(A_{2 v}\right) S B_{3 v}^{-A_{3 v}} B_{1 v}^{-A_{1 v}} B_{2 v}^{-A_{2 v}} B\left(B_{0 v}+2, A_{0 v}+C_{0 v}\right) B\left(A_{0 v}, C_{0 v}\right)},
\end{aligned}
$$

where $v=1$ for UP and for JP $v=2$.

\section{Limiting Expressions}

In regard to the uncensored sampling scheme, limiting expressions have wide applications. When test termination time $t \longrightarrow \infty$, it can be observed that uncensored points $r$ approach $n$ (sample size) and $r_{l}$ belongs to $n_{l}$, where $l=1, \ldots$, 3. The points that are censored have turned out to be uncensored then and information given in the sample has also raised here. As a result, the effectiveness of the BEs is also raised due to the consideration of all the points in sample. Thus, limiting expressions for the NIPs can easily be obtained.

The limiting forms under UP and JP for the BEs and PRs are reported in Table 1.

\section{Simulation Study}

Simulation analysis is designed to check the presentation of BEs under UP and JP and with four different LFs for the 3component mixture model of the EIWD:

(i) At the first step, we have generated several sample sizes $n=50,100,200$, and 500 assuming the different parametric values fixed as $\left(\theta_{1}, \theta_{2}, \theta_{3}\right.$, $\left.w_{1}, w_{2}\right)=\{(4,3,2,0.5,0.3),(3,3,3,0.4,0.4),(2,3,4$, $0.3,0.5)\}$. (ii) By using Mathematica software, outcomes are averaged, by reiteration of simulation 1000 times.

(iii) Randomly selected sample sizes $w_{1} n, w_{2} n$, and (1$\left.w_{1}-w_{2}\right) n$ belong to the first, second, and third factor densities, respectively.

(iv) The right type I censoring procedure is applied to conclude the effect of $t$ on BEs.

(v) All observations which were greater than $t$ have been considered as censored ones. Different fixed censoring times $t$ are used to assess the effect of censoring rate on the estimates.

(vi) In favor of the fixed $t$, values are taken as $t=25$ and 30.

(vii) The points those are larger than $t$ are taken as censored points.

(viii) It is noted that only failures can be identified as a member of subpopulations I, II, and III of the 3component mixture of EIWD.

In Table 2, results of simulation study are presented for $n=50,100,200,500$ and $\left(\theta_{1}, \theta_{2}, \theta_{3}, w_{1}, w_{2}\right)=(4,3,2,0.5,0.3)$.

From Table 2 , it is examined that BEs assuming all stated NIPs and LFs are larger for the small $n$ as compared to the highest $n$ for $t$. It is noted that, for $t$, the variation of the BEs from supposed components is near to zero with the rise in $n$. However, the PRs assuming the mentioned priors and LFs decrease with the rise in $n$. In regard to the estimation of 


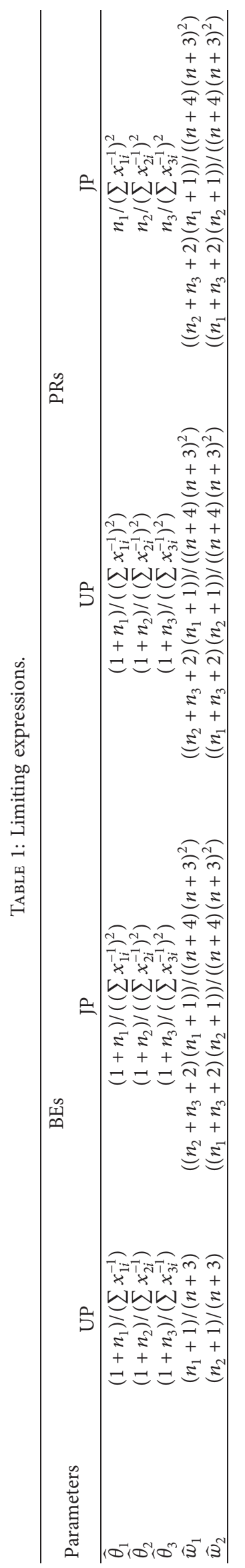


TABLE 2: Simulated results of 3-component mixture of EIWD.

\begin{tabular}{|c|c|c|c|c|c|c|c|c|c|c|c|c|c|}
\hline \multirow{2}{*}{$t$} & \multirow{2}{*}{$n$} & \multirow{2}{*}{\multicolumn{2}{|c|}{ LFs }} & \multicolumn{5}{|c|}{ UP } & \multicolumn{5}{|c|}{$\mathrm{JP}$} \\
\hline & & & & $\theta_{1}$ & $\theta_{2}$ & $\theta_{3}$ & $\widehat{w}_{1}$ & $\widehat{w}_{2}$ & $\theta_{1}$ & $\theta_{2}$ & $\theta_{3}$ & $\widehat{w}_{1}$ & $\widehat{w}_{2}$ \\
\hline \multirow{32}{*}{25} & \multirow{8}{*}{50} & \multirow{2}{*}{ SELF } & BEs & 3.463 & 3.362 & 2.965 & 0.467 & 4.364 & 2.268 & 1.948 & 0.457 & 0.313 & 0.297 \\
\hline & & & PRs & 0.675 & 0.943 & 0.978 & 0.005 & 0.908 & 0.367 & 0.379 & 0.005 & 0.004 & 0.004 \\
\hline & & \multirow{2}{*}{ QLF } & BEs & 3.526 & 2.794 & 1.182 & 0.469 & 4.149 & 2.042 & 1.255 & 0.449 & 0.266 & 0.285 \\
\hline & & & PRs & 0.041 & 0.066 & 0.111 & 0.022 & 0.052 & 0.090 & 0.125 & 0.027 & 0.061 & 0.047 \\
\hline & & & BEs & 3.627 & 2.601 & 2.450 & 0.484 & 3.736 & 3.919 & 1.411 & 0.493 & 0.286 & 0.312 \\
\hline & & PLF & PRs & 0.177 & 0.176 & 0.228 & 0.011 & 0.184 & 0.334 & 0.144 & 0.011 & 0.016 & 0.014 \\
\hline & & & BEs & 3.375 & 2.703 & 2.724 & 0.457 & 3.228 & 2.729 & 2.191 & 0.499 & 0.312 & 0.333 \\
\hline & & DLF & PRs & 0.045 & 0.062 & 0.083 & 0.025 & 0.043 & 0.071 & 0.100 & 0.021 & 0.046 & 0.042 \\
\hline & & & BEs & 3.756 & 2.889 & 1.884 & 0.489 & 3.510 & 2.486 & 1.461 & 0.498 & 0.318 & 0.302 \\
\hline & & SELF & PRs & 0.313 & 0.309 & 0.197 & 0.002 & 0.287 & 0.146 & 0.142 & 0.002 & 0.002 & 0.002 \\
\hline & & & BEs & 4.006 & 2.474 & 1.542 & 0.468 & 3.560 & 2.829 & 2.302 & 0.488 & 0.266 & 0.282 \\
\hline & & QLF & PRs & 0.024 & 0.040 & 0.055 & 0.012 & 0.022 & 0.041 & 0.055 & 0.011 & 0.029 & 0.028 \\
\hline & 100 & PI F & BEs & 3.394 & 2.720 & 1.607 & 0.446 & 3.574 & 2.792 & 1.777 & 0.496 & 0.296 & 0.337 \\
\hline & & PLF & PRs & 0.083 & 0.083 & 0.077 & 0.006 & 0.081 & 0.108 & 0.094 & 0.005 & 0.007 & 0.007 \\
\hline & & & BEs & 4.483 & 3.024 & 1.585 & 0.474 & 2.866 & 2.905 & 2.972 & 0.463 & 0.326 & 0.314 \\
\hline & & DLF & PRs & 0.037 & 0.037 & 0.050 & 0.013 & 0.023 & 0.033 & 0.047 & 0.012 & 0.021 & 0.025 \\
\hline & & & BEs & 3.572 & 2.758 & 1.785 & 0.494 & 3.299 & 3.383 & 1.662 & 0.517 & 0.293 & 0.306 \\
\hline & & SELF & PRs & 0.141 & 0.138 & 0.091 & 0.001 & 0.125 & 0.233 & 0.089 & 0.001 & 0.001 & 0.001 \\
\hline & & & BEs & 3.618 & 2.362 & 1.468 & 0.492 & 3.462 & 2.994 & 2.198 & 0.479 & 0.299 & 0.261 \\
\hline & & QLF & PRs & 0.011 & 0.021 & 0.025 & 0.005 & 0.011 & 0.018 & 0.029 & 0.006 & 0.012 & 0.016 \\
\hline & 200 & & BEs & 3.408 & 2.987 & 2.025 & 0.503 & 3.839 & 2.731 & 1.712 & 0.472 & 0.305 & 0.306 \\
\hline & & PLF & PRs & 0.035 & 0.051 & 0.055 & 0.002 & 0.045 & 0.050 & 0.043 & 0.002 & 0.003 & 0.003 \\
\hline & & & BEs & 3.241 & 3.174 & 1.715 & 0.485 & 3.554 & 2.713 & 2.162 & 0.499 & 0.284 & 0.311 \\
\hline & & DLF & PRs & 0.011 & 0.017 & 0.025 & 0.005 & 0.011 & 0.020 & 0.025 & 0.005 & 0.014 & 0.012 \\
\hline & & & BEs & 3.972 & 2.448 & 1.853 & 0.507 & 3.365 & 2.956 & 1.827 & 0.478 & 0.303 & 0.292 \\
\hline & & SELF & PRs & 0.053 & 0.046 & 0.039 & 0.000 & 0.055 & 0.067 & 0.035 & 0.000 & 0.000 & 0.000 \\
\hline & & & BEs & 4.151 & 2.686 & 1.730 & 0.481 & 3.916 & 2.462 & 2.296 & 0.483 & 0.300 & 0.303 \\
\hline & & QLF & PRs & 0.004 & 0.007 & 0.010 & 0.002 & 0.004 & 0.007 & 0.010 & 0.002 & 0.005 & 0.005 \\
\hline & 500 & & BEs & 4.039 & 2.360 & 1.852 & 0.489 & 3.167 & 2.732 & 1.910 & 0.472 & 0.308 & 0.300 \\
\hline & & PLF & PRs & 0.016 & 0.017 & 0.019 & 0.001 & 0.015 & 0.019 & 0.019 & 0.001 & 0.001 & 0.001 \\
\hline & & DLF & BEs & 3.090 & 2.527 & 1.874 & 0.490 & 3.208 & 2.697 & 2.150 & 0.475 & 0.311 & 0.291 \\
\hline & & DLF & PRs & 0.004 & 0.007 & 0.010 & 0.002 & 0.004 & 0.007 & 0.010 & 0.002 & 0.004 & 0.005 \\
\hline & & & BEs & 3.258 & 1.916 & 1.648 & 0.448 & 3.454 & 2.112 & 2.335 & 0.479 & 0.300 & 0.326 \\
\hline & & SELF & PRs & 0.531 & 0.262 & 0.302 & 0.004 & 0.518 & 0.318 & 0.545 & 0.004 & 0.004 & 0.004 \\
\hline & & OIF & BEs & 4.768 & 3.668 & 1.285 & 0.488 & 4.129 & 1.896 & 1.281 & 0.436 & 0.291 & 0.276 \\
\hline & & QLF & PRs & 0.041 & 0.071 & 0.125 & 0.021 & 0.047 & 0.071 & 0.111 & 0.025 & 0.047 & 0.051 \\
\hline & 50 & & BEs & 4.479 & 2.845 & 1.481 & 0.453 & 3.424 & 3.178 & 2.080 & 0.484 & 0.306 & 0.333 \\
\hline & & PLF & PRs & 0.197 & 0.169 & 0.126 & 0.010 & 0.014 & 0.215 & 0.193 & 0.010 & 0.013 & 0.013 \\
\hline & & & BEs & 4.752 & 1.941 & 1.233 & 0.497 & 2.472 & 3.922 & 2.253 & 0.490 & 0.320 & 0.327 \\
\hline & & DLF & PRs & 0.043 & 0.066 & 0.100 & 0.022 & 0.040 & 0.062 & 0.090 & 0.019 & 0.040 & 0.045 \\
\hline & & & BEs & 3.429 & 2.368 & 1.760 & 0.460 & 4.626 & 3.095 & 2.477 & 0.504 & 0.290 & 0.318 \\
\hline & & SELF & PRs & 0.294 & 0.207 & 0.172 & 0.002 & 0.465 & 0.368 & 0.341 & 0.002 & 0.002 & 0.002 \\
\hline & & & BEs & 3.627 & 2.506 & 2.094 & 0.466 & 4.108 & 2.484 & 1.380 & 0.467 & 0.300 & 0.293 \\
\hline & 100 & QLF & PRs & 0.022 & 0.035 & 0.052 & 0.012 & 0.023 & 0.037 & 0.058 & 0.012 & 0.024 & 0.025 \\
\hline & 100 & & BEs & 3.556 & 2.760 & 1.992 & 0.491 & 3.148 & 3.067 & 2.737 & 0.491 & 0.322 & 0.305 \\
\hline & & PLF & PRs & 0.074 & 0.092 & 0.096 & 0.005 & 0.068 & 0.103 & 0.153 & 0.005 & 0.007 & 0.007 \\
\hline & & & BEs & 3.436 & 2.850 & 1.987 & 0.467 & 3.836 & 4.765 & 1.836 & 0.489 & 0.319 & 0.319 \\
\hline 20 & & DLF & PRs & 0.022 & 0.033 & 0.045 & 0.012 & 0.020 & 0.032 & 0.050 & 0.010 & 0.021 & 0.022 \\
\hline 30 & & & BEs & 4.025 & 2.616 & 1.705 & 0.476 & 3.419 & 2.356 & 1.824 & 0.499 & 0.311 & 0.300 \\
\hline & & SELF & PRs & 0.193 & 0.131 & 0.076 & 0.001 & 0.131 & 0.100 & 0.100 & 0.001 & 0.001 & 0.001 \\
\hline & & & BEs & 3.383 & 3.007 & 2.260 & 0.505 & 4.008 & 3.137 & 2.204 & 0.483 & 0.293 & 0.285 \\
\hline & & QLF & PRs & 0.010 & 0.018 & 0.028 & 0.005 & 0.011 & 0.018 & 0.027 & 0.005 & 0.012 & 0.013 \\
\hline & 200 & PIF & BEs & 3.478 & 3.146 & 2.066 & 0.046 & 3.304 & 2.595 & 2.484 & 0.480 & 0.312 & 0.317 \\
\hline & & PLF & PRs & 0.042 & 0.056 & 0.053 & 0.003 & 0.040 & 0.049 & 0.069 & 0.003 & 0.003 & 0.003 \\
\hline & & & BEs & 3.215 & 3.003 & 2.155 & 0.462 & 3.562 & 3.297 & 2.152 & 0.477 & 0.311 & 0.327 \\
\hline & & DLF & PRs & 0.012 & 0.017 & 0.025 & 0.006 & 0.011 & 0.018 & 0.025 & 0.006 & 0.012 & 0.011 \\
\hline & & SEIF & BEs & 3.285 & 2.872 & 1.806 & 0.480 & 3.968 & 2.779 & 2.162 & 0.487 & 0.302 & 0.311 \\
\hline & & SELF & PRs & 0.049 & 0.059 & 0.035 & 0.000 & 0.049 & 0.057 & 0.050 & 0.000 & 0.000 & 0.000 \\
\hline & & & BEs & 3.834 & 2.763 & 1.823 & 0.479 & 3.917 & 2.854 & 2.087 & 0.491 & 0.300 & 0.300 \\
\hline & & QLF & PRs & 0.004 & 0.007 & 0.010 & 0.002 & 0.004 & 0.007 & 0.011 & 0.002 & 0.004 & 0.005 \\
\hline & 500 & PI F & BEs & 3.697 & 2.874 & 2.149 & 0.473 & 3.741 & 2.589 & 1.838 & 0.481 & 0.313 & 0.309 \\
\hline & & PLF & PRs & 0.017 & 0.021 & 0.022 & 0.001 & 0.017 & 0.018 & 0.019 & 0.001 & 0.001 & 0.001 \\
\hline & & & BEs & 3.601 & 2.663 & 1.858 & 0.493 & 3.424 & 2.864 & 1.990 & 0.493 & 0.302 & 0.294 \\
\hline & & DLF & PRs & 0.004 & 0.007 & 0.010 & 0.002 & 0.004 & 0.007 & 0.010 & 0.002 & 0.005 & 0.005 \\
\hline
\end{tabular}


component part of the parameters, PRs give smaller results among the DLF, over to the results mentioned among the SELF, QLF, and PLF at different $n$ and $t$. For proportion parameter estimation, it is reported that SELF illustrates minimum PRs compared to QLF, then PLF, and at last DLF. Therefore, results indicate that JP is the most suited prior over the UP for this study. On the ground of simulation formulation under the studied LFs, the DLF is considered the best for evaluating the component part of the parameters and SELF is observed to be efficient for proportion parameters.

\section{Practical Application}

EIWD has extensive uses in the area of tensile strength of carbon fiber. Therefore, in this view of analysis, we have used the data information of 100 sample points of tensile strength of carbon fiber. Earlier, this dataset is also studied by [22], which later has been considered by [5]. This dataset is based on the tensile strength of 100 observations of carbon fiber and is as follows: 3.7, 3.11, 4.42, 3.28, 3.75, 2.96, 3.39, 3.31, $3.15,2.81,1.41,2.76,3.19,1.59,2.17,3.51,1.84,1.61,1.57$, $1.89,2.74,3.27,2.41,3.09,2.43,2.53,2.81,3.31,2.35,2.77$, $2.68,4.91,1.57,2.00,1.17,2.17,0.39,2.79,1.08,2.88,2.73$, $2.87,3.19,1.87,2.95,2.67,4.20,2.85,2.55,2.17,2.97,3.68$, $0.81,1.22,5.08,1.69,3.68,4.70,2.03,2.82,2.50,1.47,3.22$, $3.15,2.97,2.93,3.33,2.56,2.59,2.83,1.36,1.84,5.56,1.12$, $2.48,1.25,2.48,2.03,1.61,2.05,3.60,3.11,1.69,4.90,3.39$, $3.22,2.55,3.56,2.38,1.92,0.98,1.59,1.73,1.71,1.18,4.38$, $0.85,1.80,2.12$, and 3.65 .

To demonstrate the proposed methodology, we have braked the given uncensored dataset in the 3-component parts according to right type I censoring scheme with rate of failure $r=91$. It is unidentified which parametric part fails till a failure happens at or before $t=9$. Having run the program in Mathematica software, the total tests are formulated 100 times:

$$
\begin{aligned}
n & =100, \\
\sum_{k=1}^{r_{1}} x_{1 k} & =5.98, \\
\sum_{k=1}^{r_{2}} x_{2 k} & =4.14, \\
\sum_{k=1}^{r_{3}} x_{3 k} & =3.09, \\
n-r & =9, \\
r_{1} & =46, \\
r_{2} & =27, \\
r_{3} & =18, \\
r & =91 .
\end{aligned}
$$

\begin{tabular}{|c|c|c|c|c|c|c|c|}
\hline Prior & LF & & $\theta_{1}$ & $\theta_{2}$ & $\theta_{3}$ & $\widehat{w}_{1}$ & $\widehat{w}_{2}$ \\
\hline \multirow{8}{*}{ UP } & \multirow{2}{*}{ SELF } & BEs & 8.53512 & 7.48614 & 6.79375 & 0.49514 & 0.30097 \\
\hline & & PRs & 1.42860 & 1.80782 & 2.19786 & 0.00244 & 0.00202 \\
\hline & \multirow{2}{*}{ QLF } & BEs & 8.20041 & 7.00317 & 6.14672 & 0.48514 & 0.28312 \\
\hline & & PRs & 0.02000 & 0.03300 & 0.05000 & 0.01029 & 0.02376 \\
\hline & \multirow{2}{*}{ PLF } & BEs & 8.61839 & 7.60597 & 6.95362 & 0.49757 & 0.30431 \\
\hline & & PRs & 0.16654 & 0.23957 & 0.31975 & 0.00484 & 0.00668 \\
\hline & \multirow{2}{*}{ DLF } & BEs & 8.70247 & 7.72763 & 7.11726 & 0.50000 & 0.30769 \\
\hline & & PRs & 0.01923 & 0.03125 & 0.04545 & 0.00971 & 0.02184 \\
\hline \multirow{8}{*}{$J P$} & \multirow{2}{*}{ SELF } & BEs & 8.36776 & 7.24466 & 6.47024 & 0.49514 & 0.30097 \\
\hline & & PRs & 1.40039 & 1.74950 & 2.09320 & 0.00240 & 0.00202 \\
\hline & \multirow{2}{*}{ QLF } & BEs & 8.03305 & 6.76168 & 5.82321 & 0.48515 & 0.28713 \\
\hline & & PRs & 0.02041 & 0.03448 & 0.05263 & 0.01029 & 0.02376 \\
\hline & \multirow{2}{*}{ PLF } & BEs & 8.45102 & 7.36441 & 6.63002 & 0.49757 & 0.30431 \\
\hline & & PRs & 0.16653 & 0.23951 & 0.31957 & 0.00484 & 0.00668 \\
\hline & \multirow{2}{*}{ DLF } & BEs & 8.53512 & 7.48614 & 6.79375 & 0.50000 & 0.30769 \\
\hline & & PRs & 0.01960 & 0.03226 & 0.04762 & 0.00971 & 0.02184 \\
\hline
\end{tabular}

Almost, here 9\% censored observations are used. BEs and the PRs assuming the NIPs are reported in Table 3.

From Table 3, it is noted that simulated results are compatible to real data study. There seem to be a few
TABLE 3: Real data results of 3-component mixture of EIWD.

exceptions which are the reasons of the small set of data information. Both simulation and real data application results among the JP are the most accurate compared to the results under UP. However, DLF in contrast to other three LFs (SELF, QLF, and PLF) illustrates enhanced findings for the proportional part of parameters.

\section{Conclusion}

In this paper, the Bayesian formulation of the 3-component mixture model of EIWD under right type I censoring technique is studied. The comprehensive simulation process is built to evaluate and demonstrate several significant features about the BEs of the 3-component mixture model of EIWD assuming the NIPs under the different symmetric and asymmetric LFs (SELF, PLF, DLF, and QLF). Overestimation and underestimation of mixture proportion are inversely related to the sample size and are directly proportional to censoring rate. A small sample size and a large censoring rate cause the higher level of overestimation. But this effect can be reduced by using a large sample size. Posterior densities are derived and notified that they are in closed forms. The second aim of this paper was the selection of appropriate LF and prior for the inference of mixture parameters at different $n$ and $t$. To judge the performance, we derived different posterior summaries, like BEs and their respective PRs by assuming different $n$ and $t$. The limiting terms for the BEs and PRs of the shape parameters which are unknown here are also obtained among the said LFs (SELF, QLF, DLF, and PLF) assuming the NIPs (JP and UP). The compatible results are observed for simulation and real dataset analysis, to evaluate the performance of BEs. The contact of several $n$ and $t$ is estimated for BEs. The outcomes revealed that, for the component parameters, the order of best $\mathrm{BEs}$ is as follows: $\mathrm{DLF}<\mathrm{PLF}<\mathrm{SELF}<\mathrm{QLF}$, and on behalf of the proportion part of parameters, this categorizing is observed as SELF $<\mathrm{PLF}<\mathrm{DLF}<\mathrm{QLF}$. Thus, we conclude that the efficient and most preferable prior is JP over the UP due to the reason of minimum risk. DLF performed better 
for 3-component mixture of EIWD to the PLF, SELF, and QLF.

\section{Data Availability}

The quantitative data related to carbon tensile strength used to support the findings of this study are included within the article.

\section{Conflicts of Interest}

The authors declare that they have no conflicts of interest.

\section{Acknowledgments}

The authors are very grateful to the Deanship of Scientific Research at King Khalid University, Kingdom of Saudi Arabia, for funding this work through research groups program under Project no. RGP-2/67/41.

\section{References}

[1] A. N. Cheema and M. Aslam, "Bayesian analysis for 3component mixture of exponentiated weibull distribution assuming non-informative priors," Journal of Statistical Computation and Simulation, vol. 90, no. 4, pp. 586-605, 2020.

[2] W.-K. Pang, S.-H. Hou, and W.-T. Yu, "On a proper way to select population failure distribution and a stochastic optimization method in parameter estimation," European Journal of Operational Research, vol. 177, no. 1, pp. 604-611, 2007.

[3] A. Aljuaid, "Estimating the parameters of an exponentiated inverted weibull distribution under type-II censoring," Applied Mathematical Sciences, vol. 7, no. 35, pp. 1721-1736, 2013.

[4] I. Gupta, "Estimation of parameter and reliability function of exponentiated inverted weibull distribution using classical and bayesian approach," International Journal of Recent Scientific Research, vol. 8, no. 7, pp. 18117-18819, 2017.

[5] A. Flaih, H. Elsalloukh, E. Mendi, and M. Milanova, "The exponentiated inverted weibull distribution," Applied Mathematics \& Information Sciences, vol. 6, no. 2, pp. 167-171, 2012.

[6] A. Ahmad, S. P. Ahmad, and A. Ahmed, "Bayesian estimation of exponentiated inverted weibull distribution under asymmetric loss function," Journal of Statistic Applications \& Probability, vol. 4, no. 1, pp. 183-192, 2015.

[7] I. Gupta and R. Gupta, "Bayesian and E-Bayesian estimation of the unknown shape parameter of exponentiated inverted weibull distribution using different loss functions," International Educational Scientific Research Journal, vol. 3, no. 5, pp. 408-416, 2017.

[8] A. Saghir, S. Tazeem, and I. Ahmad, "The weighted exponentiated webill distribution: properties and application," Journal of Informatics and Mathematical Sciences, vol. 9, no. 1, pp. 137-151, 2017.

[9] M. Tahir, M. Aslam, and Z. Hussain, "Estimation of parameters of the 3-component mixture of pareto distributions using type-I right censoring under Bayesian paradigm," Journal of the National Science Foundation of Sri Lanka, vol. 44, no. 3, pp. 327-343, 2016.

[10] M. Aslam, M. Tahir, Z. Hussain, and B. Al-Zahrani, "A 3component mixture of Rayleigh distributions: properties and estimation in Bayesian framework," PLoS One, vol. 10, no. 5, Article ID e0126183, 2015.

[11] M. Tahir, M. Aslam, and Z. Hussain, "On the Bayesian analysis of 3-componen mixture of exponential distribution under different loss functions," Hacettepe Journal of Mathematics and Statistics, vol. 45, no. 51, pp. 609-628, 2016.

[12] M. Tahir, M. Aslam, Z. Hussain, and N. Abbas, "On the finite mixture of exponential, Rayleigh and burr type-XII distributions: estimation of parameters in Bayesian framework," Electronic Journal of Applied Statistical Analysis, vol. 10, no. 1, pp. 271-293, 2017.

[13] P. S. de Laplace, Theorie Analytique Des Probabilities, Courair, Paris, France, 1820.

[14] T. Bayes, "An essay toward solving a problem in the doctrine of chances," Philosophical Transactions of the Royal Society of London, vol. 53, pp. 370-418, 1763.

[15] S. Geisser, "On prior distributions for binary trials," The American Statistician, vol. 38, no. 4, pp. 244-247, 1984.

[16] J. M. Bernardo, "Reference posterior distributions for Bayesian inference," Journal of the Royal Statistical Society: Series B (Methodological), vol. 41, no. 2, pp. 113-128, 1979.

[17] H. Jeffreys, "An invariant form for the prior probability in estimation problems," Proceedings of the Royal Society of London. Series A, Mathematical and Physical Sciences, vol. 186, no. 1007, pp. 453-461, 1946.

[18] H. Jeffreys, Theory of Probability, Claredon Press, Oxford, UK, 1961.

[19] J. O. Berger, Statistical Decision Theory and Bayesian Analysis, Springer-Verlag, New York, NY, USA, 2nd edition, 1985.

[20] J. G. Norstrom, "The use of precautionary loss functions in risk analysis," IEEE Transactions on Reliability, vol. 45, no. 3, pp. 400-403, 1996.

[21] M. H. DeGroot, Optimal Statistical Decision, McGraw-Hill, New York, NY, USA, 2005.

[22] M. D. Nichols and W. J. Padgett, "A bootstrap control chart for weibull percentiles," Quality and Reliability Engineering International, vol. 22, no. 2, pp. 141-151, 2006. 